\title{
Bacterial virulence factor inhibits caspase-4/11 activation in intestinal epithelial cells
}

\author{
MA Pallett ${ }^{1}$, VF Crepin ${ }^{1}$, N Serafini ${ }^{2,3}$, M Habibzay ${ }^{1}$, O Kotik ${ }^{1}$, J Sanchez-Garrido ${ }^{4}$, JP Di Santo ${ }^{2,3}$, \\ AR Shenoy ${ }^{4}, \mathrm{CN}$ Berger $^{1}$ and G Frankel ${ }^{1}$
}

The human pathogen enteropathogenic Escherichia coli (EPEC), as well as the mouse pathogen Citrobacter rodentium, colonize the gut mucosa via attaching and effacing lesion formation and cause diarrheal diseases. EPEC and C. rodentium type III secretion system (T3SS) effectors repress innate immune responses and infiltration of immune cells. Inflammatory caspases such as caspase-1 and caspase-4/11 are crucial mediators of host defense and inflammation in the gut via their ability to process cytokines such as interleukin (IL)-1ß and IL-18. Here we report that the effector NleF binds the catalytic domain of caspase- 4 and inhibits its proteolytic activity. Following infection of intestinal epithelial cells (IECs) EPEC inhibited caspase-4 and IL-18 processing in an NleF-dependent manner. Depletion of caspase-4 in IECs prevented the secretion of mature IL-18 in response to infection with EPECAnleF. NleF-dependent inhibition of caspase-11 in colons of mice prevented IL-18 secretion and neutrophil influx at early stages of $C$. rodentium infection. Neither wild-type $C$. rodentium nor $C$. rodentium $\Delta$ nleF triggered neutrophil infiltration or IL-18 secretion in Cas11 or Casp1/11-deficient mice. Thus, IECs have a key role in modulating early innate immune responses in the gut via a caspase-4/11-IL-18 axis, which is targeted by virulence factors encoded by enteric pathogens.

\section{INTRODUCTION}

Central to the infection strategy of the extracellular pathogens enteropathogenic Escherichia coli (EPEC), enterohaemorrhagic E. coli (EHEC) $)^{1}$ and Citrobacter rodentium ${ }^{2}$ is injection of type III secretion system effectors into intestinal epithelial cells (IECs) where they target diverse signaling pathways, particularly innate immune signaling. $\mathrm{NleC}$ and $\mathrm{NleD}$ are $\mathrm{Zn}$ dependent endopeptidases that specifically cleave and disable RelA (p65) and JNK, respectively, thus blocking NF- $\kappa B$ and AP-1 activation. ${ }^{3} \mathrm{NleE}$ is a methyltransferase that specifically modifies a cysteine in the zinc finger domain of TAB2 and TAB3, thus also blocking NF- $\kappa B$ signaling. ${ }^{4} \mathrm{NleB}$, which also inhibits NF- $\kappa \mathrm{B}$, has an $\mathrm{N}$-acetylglucosamine transferase activity that specifically modifies Arg 117 in the death domain of $\mathrm{FADD}^{5,6}$ and $\mathrm{NleH}$ is a serine/threonine kinase that inhibits the RPS3/NF- $\kappa B$ pathway via phosphorylation of CRKL (v-Crk sarcoma virus CT10 oncogene-like protein). ${ }^{7}$
Inhibition of innate immunity by EPEC and EHEC is needed to counter its activation by the type III secretion system (T3SS), flagellins, and lipopolysaccharides (LPS), which are readily detected by sensors and receptors in mammalian hosts. In response to infection, some sensors assemble macromolecular complexes called inflammasomes to stimulate the protease activity of caspase- 1 . The proteolytic processing and release of interleukin (IL)-1 $\beta$ and IL-18, and the induction of pyroptotic cell death triggered by caspase- 1 can prevent the establishment and spread of microbial pathogens. ${ }^{8,9}$ In addition, the single mouse caspase-11 and the related human caspase- 4 and caspase- 5 act as cytosolic receptors, which bind LPS directly via their $\mathrm{N}$-terminal caspase activation and recruitment domains (p22 domain). LPS binding induces oligomerization and auto-proteolytic activation of caspase-4/5/11 into their active p20/p10 fragments and subsequent pyroptotic lysis of bacterially infected host cells. ${ }^{10}$ In human and mouse

${ }^{1}$ Department of Life Sciences, MRC Centre for Molecular Bacteriology and Infection, Imperial College, London, UK. ${ }^{2}$ Innate Immunity Unit, Institut Pasteur, Paris, France. ${ }^{3}$ Inserm U668, Paris, France and ${ }^{4}$ Department of Medicine, MRC Centre for Molecular Bacteriology and Infection, Imperial College, London, UK. Correspondence: G Frankel (g.frankel@imperial.ac.uk)

Received 19 October 2015; accepted 1 August 2016; published online 14 September 2016. doi:10.1038/mi.2016.77 
phagocytic cells, LPS is detected by caspase-4/11, which stimulate caspase-1-dependent maturation of IL-1 $\beta$ and IL-18 via the NLRP3-ASC inflammasome. ${ }^{11-13}$ However, in IECs caspase-4/11 acts independently of NLRP 3 and caspase- 1 to directly process IL-18 and induce pyroptosis during Salmonella infection. ${ }^{14}$ Therefore, the detection of Gram-negative bacteria by IECs markedly contrasts that in myeloid cells. However, unlike Salmonella, which are intracellular pathogens, extracellular pathogens use T3SS to prevent death pathways in host cells to which they intimately adhere. ${ }^{5,6,15}$ This suggests that EPEC, EHEC, and C. rodentium might manipulate caspase-4/11 and/or inflammasome pathways in IECs.

Previous work on C. rodentium infections in mice showed that loss of inflammasome signaling-related genes such as Nlrp3, Nlrc4, Casp1, Casp11, Il1 $\beta$, and Il18 results in enhanced morbidity and inflammatory disease, whereas wild-type (WT) mice clear the pathogen within $14-21$ days. ${ }^{16,17}$ Detection of $C$. rodentium, EHEC, and EPEC in myeloid cells has also been studied previously, and a recent report identified the EPEC NleA T3SS effector protein as an inhibitor of NLRP3-caspase-1 inflammasomes. ${ }^{18}$ However, as IECs use non-canonical, NLRP3-, and caspase-1-independent mechanisms to detect bacteria, we hypothesized that EPEC and C. rodentium subvert caspase-4/11 action in IECs upon initial attachment. Here we report that bacterial T3SS effector NleF is a potent inhibitor of mammalian caspase-4/11 and thus prevents IL-18 secretion from IECs in vitro, and blocks caspase-11-IL-18 mediated neutrophil influx during infection in vivo.

\section{RESULTS}

\section{NleF binds human caspase- 4}

The highly conserved effector NleF was previously reported to bind the active site and to inhibit the activity of caspase-9, caspase-8, and caspase-4, however, whether NleF affects inflammasome signaling and the innate immune response to bacterial infection in vivo has not been tested. ${ }^{19}$ By employing a yeast-2-hybrid screen (Supplementary Table S1 online) and a direct yeast-2-hybrid assay (Figure 1a) we confirmed that human caspase- 4 is an interacting partner of EPEC NleF ( $\left.\mathrm{NleF}_{\mathrm{EPEC}}\right)$. Truncation analyses revealed an interaction between $\mathrm{NleF}_{\mathrm{EPEC}}$ and the p30 catalytic domain of caspase-4 (Figure 1b). Deletion of four C-terminal residues in $\mathrm{NleF}_{\text {EPEC }}\left(\mathrm{NleF}_{1-185 \_ \text {EPEC }}\right)$ abrogates its binding to caspase$9,{ }^{19}$ and similar defects were seen in binding to caspase-4 (Figure 1b). Mutation of the substrate-binding pocket of caspase-4 (R152A, W313A, and R314A) also abolished NleFcaspase-4 interaction (Figure 1b). To confirm that the binding is direct, the caspase- 4 p20 subunit ( $22 \mathrm{kDa}$; His tagged), p10 subunit $(10 \mathrm{kDa})$, and $\mathrm{NleF}_{\mathrm{EPEC}}(65 \mathrm{kDa}$; maltose-binding protein fusion) were co-expressed, purified by tandem affinity chromatography and analyzed by gel filtration. Three chromatographic peaks corresponding to free maltose-binding protein- $\mathrm{NleF}_{\mathrm{EPEC}}$, free His-p20, and a complex containing $\mathrm{NleF}_{\mathrm{EPEC}}, \mathrm{p} 20$, and p10 subunits were observed (Figure 1c).
$\mathrm{NleF}_{\mathrm{EPEC}}$ and caspase- 4 subunits co-purified and co-eluted as a macromolecular complex with an apparent molecular weight of $\sim 230 \mathrm{kDa}$ (Figure 1c and d).

NleF inhibits human caspase- 4 and mouse caspase- 11

Recombinant caspase- 4 underwent auto-proteolytic activation presumably as a consequence of LPS binding when purified from E. coli. WT caspase-4, but not a catalytic dead mutant (caspase-4C285S), underwent auto-proteolysis to the active p20 form and hydrolyzed the caspase-4 fluorogenic substrate peptide (Ac-LEVD-AFC; Figure 2a). Recombinant $\mathrm{NleF}_{\mathrm{EPEC}}$ inhibited the activity of caspase-4 in a dose-dependent manner with an $\mathrm{IC}_{50}$ of $5 \mathrm{~nm}$ (Figure $2 \mathbf{b}$ ), comparable to $14 \mathrm{~nm}$ previously measured for $\mathrm{NleF}_{\mathrm{EHEC}}$ by Blasche et al. ${ }^{19}$ Despite not binding caspase-4 in direct yeast-2-hybrid, $\mathrm{NleF}_{1-185 \_ \text {EPEC, }}$ which was pulled down with caspase-4 at low levels (data not shown), was able to inhibit caspase-4 activity although at an $\mathrm{IC}_{50}$ of $25.5 \mathrm{~nm}$ (Figure $\left.2 \mathbf{b}\right)$. C. rodentium $\mathrm{NleF}\left(\mathrm{NleF}_{\mathrm{CR}}\right)$, which shares $84 \%$ amino-acid identity with $\mathrm{NleF}_{\mathrm{EPEC}}$, strongly inhibited the proteolytic activity of mouse caspase-11 $\left(\mathrm{IC}_{50}\right.$ of $13 \mathrm{~nm}$; Figure $2 \mathrm{c}$ and d) revealing an evolutionarily conserved functional property. Importantly, we found that $\mathrm{NleF}_{\mathrm{EPEC}}$ inhibits caspase- 4 more efficiently than $\mathrm{NleF}_{\mathrm{CR}}$ (Figure 2c), whereas $\mathrm{NleF}_{\mathrm{CR}}$ inhibits caspase-11 more efficiently than caspase-4 (Figure 2f).

NleF inhibits human caspase-4 activation during infection To investigate if $\mathrm{NleF}_{\mathrm{EPEC}}$ targets caspase- 4 during infection of human IECs, Caco- 2 cells were infected with the WT EPEC and EPEC $\Delta$ nleF; both strains adhered to the cultured cells equally (Figure 3a). However, whereas secreted caspase-4 was absent following infection with WT EPEC, the active p30 fragment of caspase- 4 was found in the supernatants of cells infected with EPEC $\Delta$ nleF (Figure $3 \mathbf{b}$ ). Addition of the caspase-4 inhibitor Ac-LEVD-CHO complemented the EPEC $\Delta$ nleF phenotype in a dose-dependent manner (Figure $\mathbf{3 b}$ ).

$\mathrm{NleF}_{\text {EPEC }}$ did not affect the expression of pro-IL-18, which was similar in uninfected cells and those infected with all the EPEC strains (Figure 3c). Although secretion of pro-IL-18 was detected upon infection with WT EPEC and EPECAnleF, proIL-18 was only processed into the active form following infection with EPECAnleF (Figure 3d). Secretion of mature IL18, induced by EPEC $\triangle n l e F$, was not detected when this strain was complemented with a plasmid encoding $\mathrm{NleF}_{\mathrm{EPEC}}$ $\left(\mathrm{pNleF}_{\mathrm{EPEC}}\right)$ (Figure 3d).

To confirm that inhibition of caspase- 4 by NleF was sufficient to block processing of IL-18, we generated Caco-2 cells depleted of caspase-4 using miRNA30E-based stable short hairpin RNA expression (Figure 4a). EPEC $\Delta n l e F$ infection of Caco-2 cells silenced for caspase-4 expression (C4) did not secrete mature IL-18, as measured by both western blotting (Figure 4b) and ELISA (enzyme-linked immunosorbent assay) (Figure 4c), clearly pointing to a requirement of caspase-4 in IL-18 processing during EPEC infection of IECs. Importantly, no cell death was detectable by measuring lactate dehydrogenase $(\mathrm{LDH})$ release or propidium iodide (PI) uptake following infection of control or caspase-4-depleted 


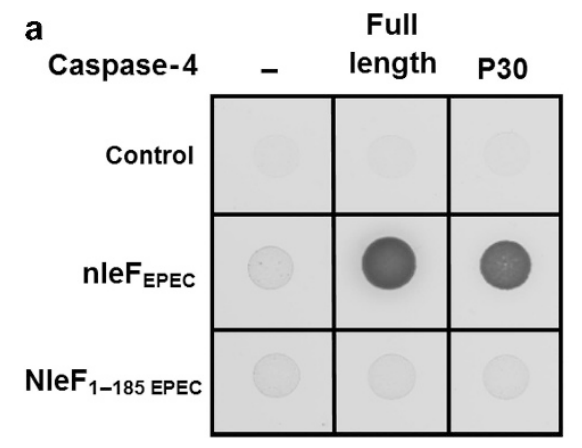

b
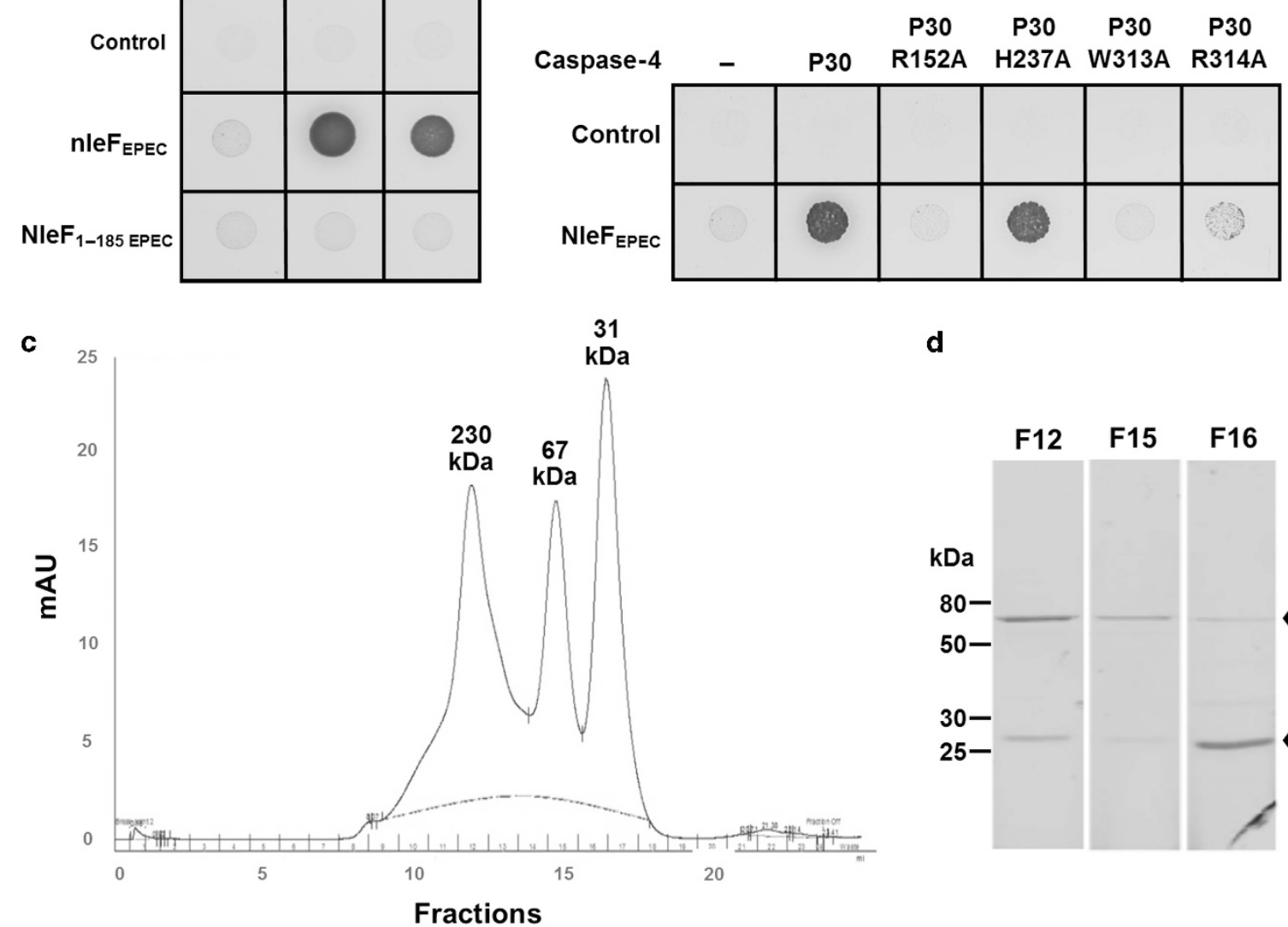

d

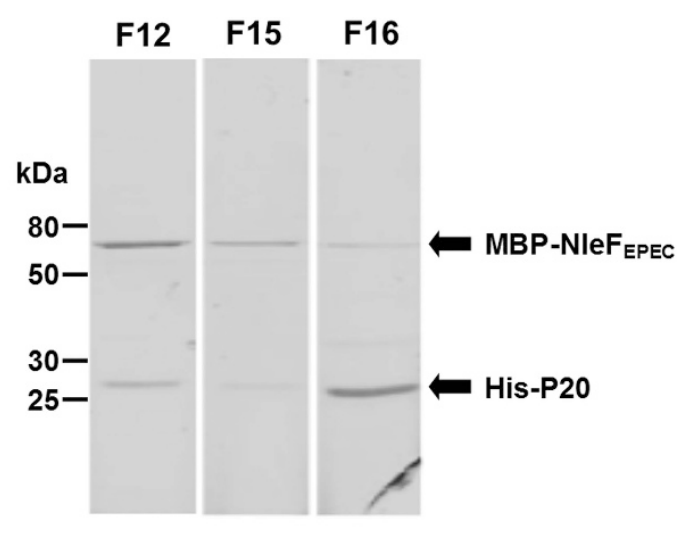

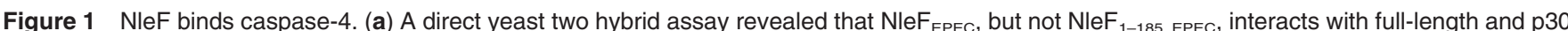
caspase-4. (b) Substitution of amino acids R152A, W313A, and R314A within the putative caspase-4 substrate domain abrogated the interaction with $\mathrm{NleF}_{\text {EPEC. }}$ (c) Fractions of size exclusion of the chromatographic profile of maltose-binding protein-N/eF $F_{E P E C}$ and His-p20/p10 caspase-4 purified by amylose and talon affinity chromatography and (d) analyzed by SDS-PAGE gel electrophoresis, revealed that NleF $F_{\text {EPEC }}$ and caspase-4 subunits copurified and co-eluted as a macromolecular complex at a MW of $\sim 230 \mathrm{kDa}$.

Caco-2 cells (Figure 4d); this is likely due to EPEC T3SS effectors (e.g., NleH, NleB), which inhibit cell death. ${ }^{5,6,15}$ Thus, in human IECs, pro-IL-18 processing during EPEC infection is caspase-4 dependent and the bacterially injected NleF specifically inhibits this process.

\section{C. rodentium inhibits IL-18 secretion in vivo in an $\mathrm{NleF}_{\mathrm{CR} \text {-dependent manner }}$}

To test the role of NleF during infection in vivo we infected C57BL/6 mice with WT C. rodentium, C. rodentium $\Delta$ nleF, or C. rodentium $\Delta n l e F$ complemented with pnle $F_{\mathrm{CR}}$. Colonization (Figure 5a) and colonic crypt hyperplasia (Figure 5b) were similar between the different $C$. rodentium strains (Figure 5). We quantified levels of IL-18 and IL- $1 \beta$ secreted from colonic explants, and the inflammasome-independent chemokine CXCL1 as a control, on days 4 and 8 post infection. On day 4 post infection of C57BL/6 mice with $C$. rodentium $\Delta n l e F$ we detected a significantly increased colonic secretion of IL-18, whereas mock-infected (phosphate-buffered saline, PBS) or WT C. rodentium-infected colons released similarly low levels of IL-18 (Figure 5c). Complementing the C. rodentium $\Delta n l e F$ mutant with a plasmid encoding $\mathrm{NleF}_{\mathrm{CR}}$ restored the inhibition of IL-18 secretion (Figure 5c); secreted IL-1 $\beta$ was below the detectable limit (data not shown). Secretion of CXCL1 was similar in colons extracted from mice treated with PBS or infected with WT $C$. rodentium or $C$. rodentium $\Delta$ nleF (Figure 5d). Complementing the $C$. rodentium $\Delta n l e F$ mutant with a plasmid encoding $\mathrm{NleF}_{\mathrm{CR}}$ resulted in a significantly increased CXCL1 secretion (Figure 5d), which is consistent with our recent finding that over expression of $\mathrm{NleF}_{\mathrm{EPEC}}$ activates NF- $\mathrm{KB}$ in cultured cells. ${ }^{20}$ Importantly, NleF-dependent inhibitory effects were only observed early during infection (day 4 post infection), and IL-18 secretion was similar following WT C. rodentium or C. rodentium $\Delta$ nleF infection on day 8 post infection (Figure 5e).

To validate that $\mathrm{NleF}_{C R}$ inhibits IL-18 secretion via the inflammasomes, we first infected Casp1/11-deficient mice with C. rodentium and C. rodentium $\Delta$ nleF. As expected, loss of Casp1 and Casp11 abolished IL-18 secretion from colonic explants after infection with WT C. rodentium or C. rodentium $\Delta n l e F$ (Figure 5c); CXCL1 secretion was similar in Casp1/11 ${ }^{-/-}$ mice infected with the two strains (data not shown). In order to 

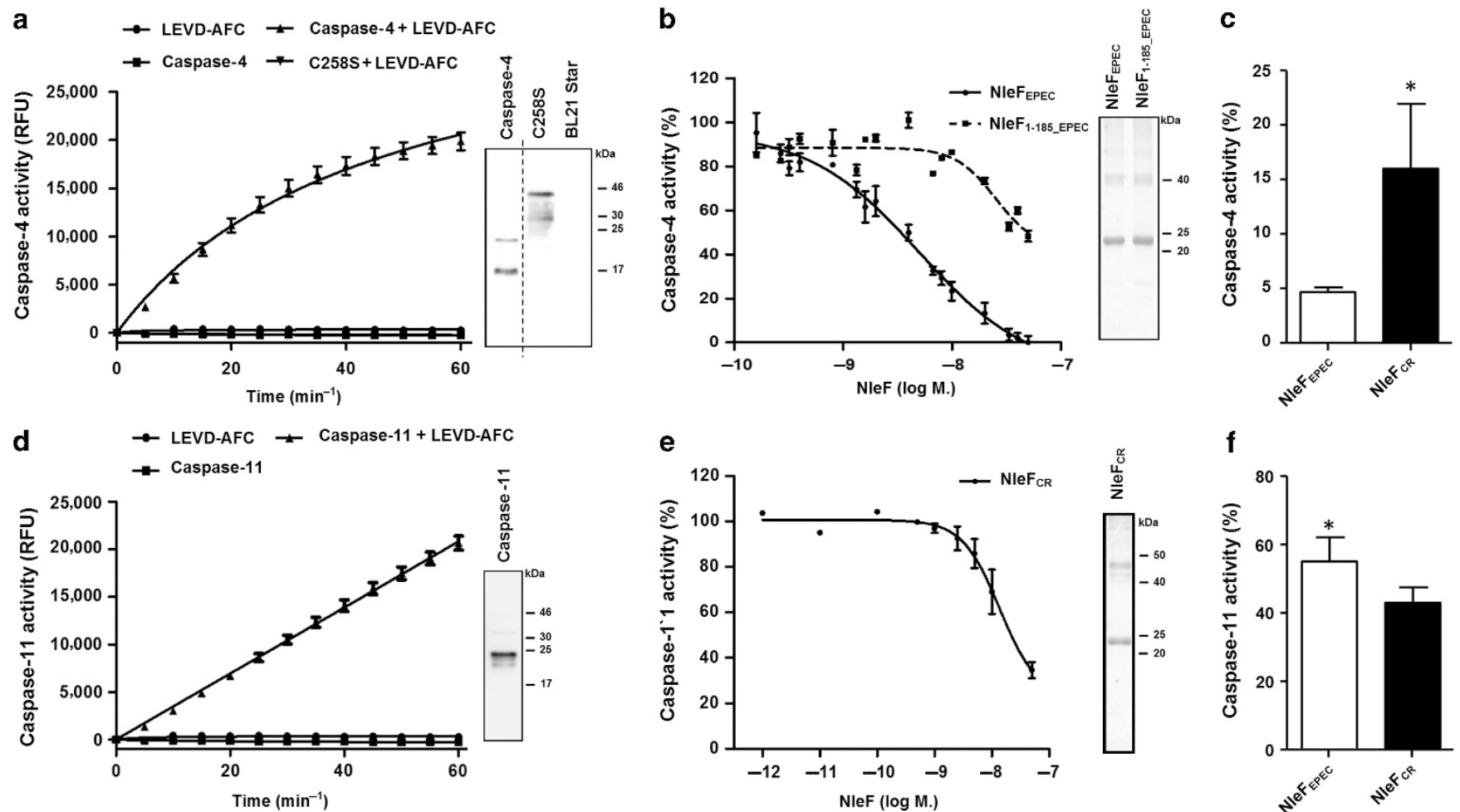

Figure 2 NleF inhibits caspase-4 activity. (a) Recombinant caspase-4, but not caspase- $4{ }^{\mathrm{C} 258 \mathrm{~S}}$, is auto-activated (western blot) and cleaves the reporter Ac-LEVD-AFC. Results are plotted as relative fluorescence units (RLU) minus background (No Ac-LEVD-AFC) over time (min). (b) Dose-dependent inhibition of caspase-4 AC-LEVD-AFC cleavage by recombinant $\mathrm{NleF}_{\text {EPEC, }}$ and $\mathrm{NleF}_{1-185 \_E P E C}$ (shown by Coomassie stained gel). $(\mathbf{c}) \mathrm{NleF}_{\text {EPEC }}\left(10 \mathrm{~nm}_{\text {) }}\right.$ inhibits the activity of caspase- 4 more efficiently than NleF $_{C R}(10 \mathrm{~nm})$ after 30 min incubation in the presence of Ac-LEVD-AFC. (d) Recombinant caspase11 is auto-activated (western blot) and cleaves the reporter Ac-LEVD-AFC. (e) Dose-dependent inhibition of caspase-11 activity by recombinant NleF ${ }_{C R}$ (shown by Coomassie stained gel). (f) $\mathrm{NleF}_{\mathrm{CR}}(50 \mathrm{nM})$ inhibits the activity of caspase- 11 more efficiently than NleF $\mathrm{EPEC}_{\mathrm{E}}(50 \mathrm{nM})$ after 30 min incubation in the presence of AC-LEVD-AFC. Results are expressed as a percentage of wild-type caspase-4 or caspase-11 RLU min ${ }^{-1}$ from at least two independent experiments. * indicates $P<0.05$.

confirm that the phenotype was due to caspase-11, we next infected Casp $11^{-I-}$ mice with C. rodentium or C. rodentium $\Delta n l e F$. This showed that whereas WT $C$. rodentium and C. rodentium $\Delta$ nleF colonized the Casp $11^{-/-}$mice at comparable levels (Figure 5f), secretion of IL-18 was extremely low and similar to that in Casp $1 / 11^{-/-}$mice (Figure 5c). We therefore concluded that caspase- 11 is responsible for secretion of IL-18 following infection with $C$. rodentium $\Delta$ nleF.

\section{IL-18 is essential for the recruitment of neutrophils early during $C$. rodentium infection}

As IL-18 facilitates neutrophil and leukocyte recruitment to sites of inflammation, ${ }^{21}$ we investigated the effect of $\mathrm{NleF}_{\mathrm{CR}}$ on immune cell recruitment. Infection of C57BL/6 mice for 4 days with $C$. rodentium $\Delta n l e F$ resulted in a significant increase in neutrophil recruitment in comparison with WT C. rodentiuminfected or PBS-treated mice (Figure 6b). Infection with the $C$. rodentium $\Delta n l e F$ pnleF $F_{\mathrm{CR}}$ strain restored the inhibition of neutrophil recruitment (Figure 6b). No significant differences were observed for other myeloid or lymphocyte cell type analyzed, including macrophages, innate lymphoid cells, B cells and $\mathrm{T}$ cells (data not shown). Furthermore, correlating with similar IL-18 secretion, no difference in neutrophil recruitment was observed at day 8 post infection (Figure $6 \mathrm{c}$ ), suggesting that
$\mathrm{NleF}_{\mathrm{CR}}$ has a specific role during early immune responses to $C$. rodentium. Enhanced neutrophil influx was Casp1/11 dependent; absence of these caspases abolished the increase in neutrophil recruitment during infection with $C$. rodentium $\Delta$ nleF (Figure 6b). Similar results were obtained following infection of Casp11 $11^{-/-}$mice (Figure 6b). Thus, $\mathrm{NleF}_{\mathrm{CR}}$ is a virulence factor responsible for early inhibition of the host inflammasomes, and that the inflammasome is essential for early neutrophil recruitment in response to $C$. rodentium infection.

\section{DISCUSSION}

Inflammasome-dependent cytokines and pyroptosis have important antimicrobial functions. ${ }^{8,9}$ It is therefore not surprising that pathogenic bacteria have evolved mechanisms to prevent inflammasome activation. ${ }^{22}$ For example, Yersinia uses YopK to prevent detection of its T3SS, ${ }^{22}$ and bacteria modify their LPS to evade detection by caspase- $11 .^{23}$ The Shigella flexneri effector OspC 3 sequesters caspase- 4 activity by binding the caspase- 4 p20 subunit to prevent p10 binding and oligomerization. ${ }^{24}$ Here we demonstrate that a virulence factor of A/E pathogens, NleF, targets the heterotetramer complex of caspase- 4 via its $\mathrm{C}$-terminal motif, underlining the importance of caspase-4 inhibition during the course of infection. 
a

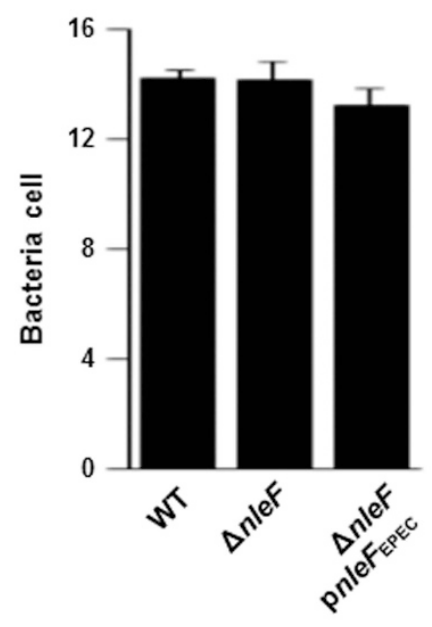

b

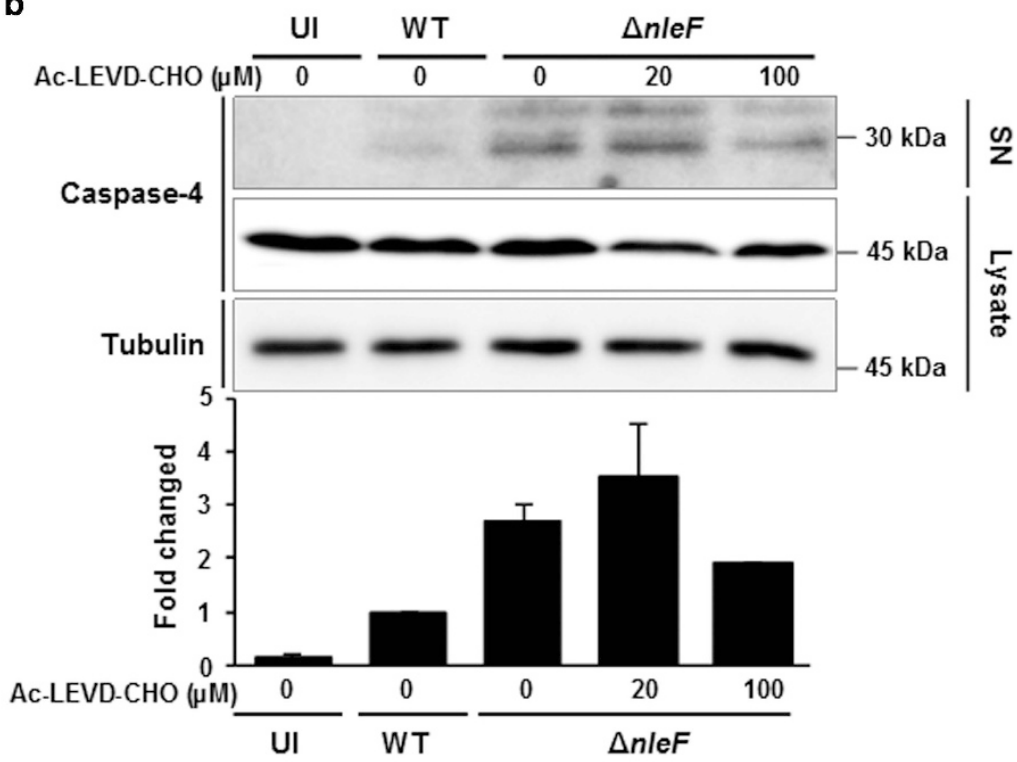

c

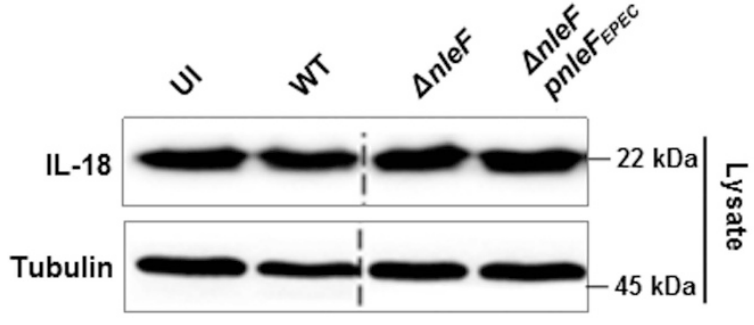

d

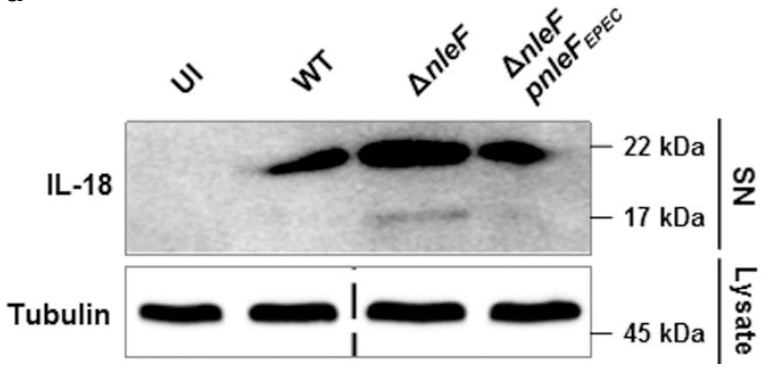

Figure 3 NleF inhibits secretion of caspase-4 and IL-18 during EPEC infection. (a) Infection of polarized Caco-2 cells with WT EPEC, EPEC $\triangle$ nleF, or the complemented strain ( $p n l e F_{E P E C}$ ) revealed similar levels of cell adhesion ( $3 \mathrm{~h}$ post infection). (b) Caco-2 cells were infected with WT EPEC or EPEC $\Delta$ nle F in the absence or presence of the inhibitor Ac-LEVD-CHO (total $21 \mathrm{~h}$ ). Immunoblotting of supernatants (SN) revealed that EPEC inhibits secretion of active caspase-4 ( $\sim 28 \mathrm{kDa}$ ) in an $\mathrm{NleF}_{\text {EPEC}}$-dependent manner, assessed by western blots (upper panel) and quantified by densitometry of multiple experiments (lower panel). (c) Infection of Caco-2 cells with WT EPEC, EPEC $\Delta$ nleF, or complemented EPEC $\Delta$ nleF $\left(p n l e F_{E P E C}\right.$ ) had no effect on the levels of total IL-18 at $4 \mathrm{~h}$ p.i. (d) NleF is essential for inhibition of IL-18 secretion from infected Caco-2 cells $(21 \mathrm{~h}$ post infection).

In agreement with our biochemical analyses, EPEC was able to inhibit caspase-4 in IECs in an NleF-dependent manner, whereas recent reports showed that infection of cultured cells with either Salmonella or EPEC led to caspase- 4 activation ${ }^{24}$ and caspase-4dependent induction of IL-18 release. ${ }^{14}$ Taken together, our data suggest that whereas EPEC can initiate caspase- 4 activation and IL-18 processing, NleF dampens this response. Previous studies have shown that Nlrp3, Nlrc4, Casp1, and Casp11 are important in protection against $C$. rodentium infection. ${ }^{16,25}$ Loss of inflammasome-related genes results in significantly increased C. rodentium bacterial load in the intestine late in infection, which may partly explain the enhanced inflammation in inflammasome-deficient mice infected with C. rodentium. Loss of inflammasome-dependent IL-1 $\beta$ and IL-18 also results in enhanced bacterial burdens at late stages of infection and susceptibility to C. rodentium infection of $I l 1 b^{-/-}$and $I l 18^{-/-}$ mice. ${ }^{16}$ Our studies establish that NleF functions at early stages of infection of mucosal surfaces by inhibiting the inflammasome and preventing release of IL-18 by epithelial cells.

We also found that $\mathrm{NleF}_{\mathrm{CR}}$ inhibited caspase-11-dependent neutrophil recruitment. IL-18 is a key regulator of the adaptive immune response, stimulates the migration of innate and adaptive immune cells, ${ }^{21,26,27}$ and controls IEC turnover and protects against damage in the intestine. ${ }^{28}$ During the early stages of infection, IL-18 is largely secreted by epithelial cells. ${ }^{17}$ Current data, ${ }^{16}$ including the secretion of IL- $1 \beta$, which is not expressed in non-hematopoietic cells, ${ }^{29}$ suggests that at later time points during C. rodentium infection colonic IL-18 secretion may switch to be myeloid cell dependent. ${ }^{16}$ Therefore, myeloid cell secretion of the IL-1 family cytokines may not be subverted by $\mathrm{NleF}_{\mathrm{CR}}$ and would become the pre-dominant source of IL-18 and IL-1 $\beta$ at the peak of infection. Similarly secretion of IL-22 is switched from innate lymphoid cells 3 at early phase of infection to IL-22-producing $\mathrm{T}$ cells at later time points $(>7$ days $) .^{30}$

The study demonstrates a pathway during infection of IECs, which leads to the activation of caspase-11, secretion of IL-18, and recruitment of neutrophil. In addition, we show that inhibition of caspase- 11 by bacterial NleF blocks this pathway in the host. Our findings are consistent with the recent study on the epithelial cell caspase-11-IL-18 axis during Salmonella infection, which reported significant neutrophil influx in 
a

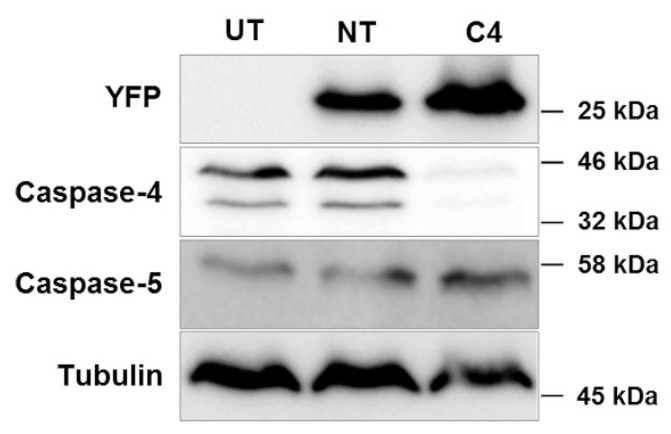

C

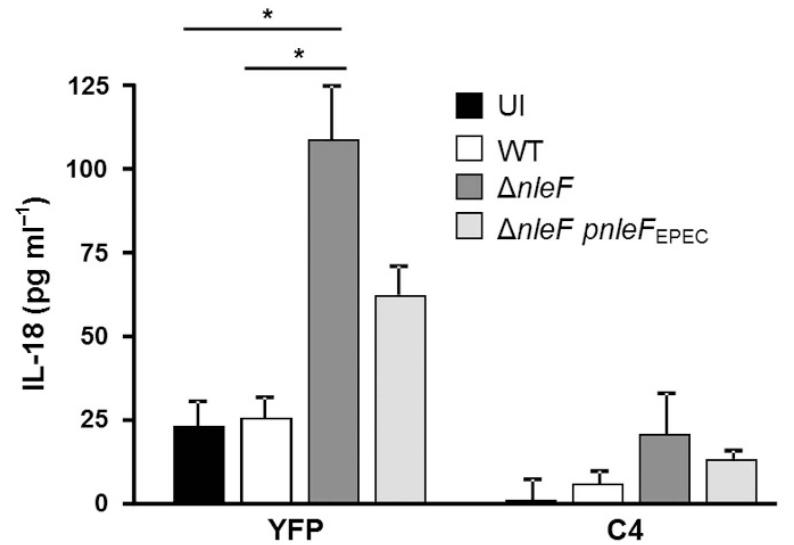

b

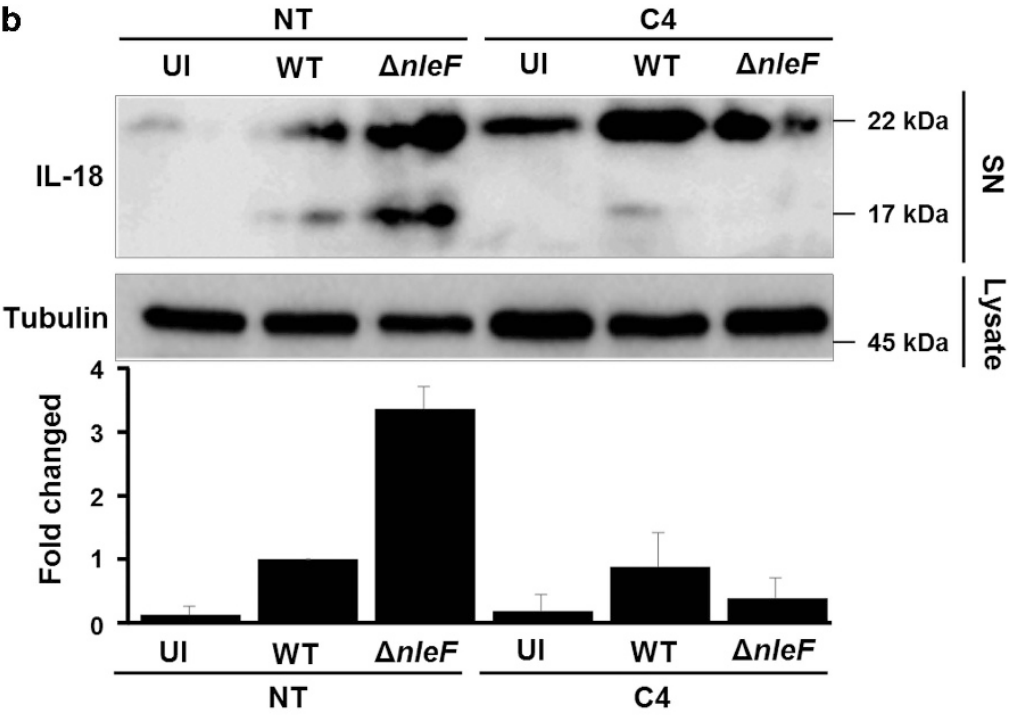

d

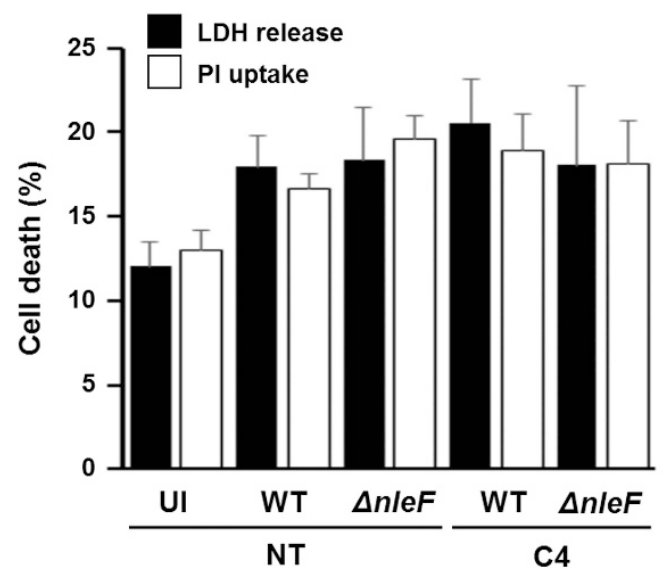

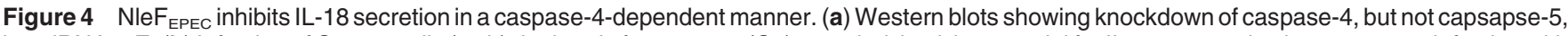
by miRNA30E. (b) Infection of Caco-2 cells $(21 \mathrm{~h})$ depleted of caspase-4 (C4) revealed that it is essential for IL-18 processing in response to infection with EPEC $\triangle \mathrm{nleF}$, assessed by western blots (upper panel) and quantified by densitometry of two independent experiments (lower panel). (c) ELISA from two biological repeats showing specific secretion of IL-18 from control (YFP), but not from C4, Caco-2 cells infected for $21 \mathrm{~h}$ with EPEC $\triangle$ nleF. (d) EPEC does not trigger LDH release or PI uptake during infection $(21 \mathrm{~h})$ of control or $\mathrm{C} 4 \mathrm{Caco}-2$ cells, results are represented as a percentage of total uptake or total release and are an average of two biological repeats carried out in triplicate. * indicates $P<0.05$.

infected gall bladder epithelia of wild-type mice, but no neutrophil influx in Casp11 $11^{-/-}$mice. $^{14,26,27,31}$

Recent studies have revealed the contribution of noninflammasome and inflammasome-forming NOD-like receptors (NLRs) in the non-hematopoietic compartment for intestinal homeostasis and the host-mediated clearance and protection against enteric pathogens. ${ }^{32}$ Mice deficient in NLRP6 have impaired goblet cell mucus exocytosis and display a microbiome-exposed epithelial cell layer and persistence of $C$. rodentium infection. ${ }^{33}$ Moreover, NLRP12 is a checkpoint for non-hematopoietic non-canonical NF- $\kappa \mathrm{B}$ activation, ${ }^{34}$ and acts as a negative regulator of colitis and colitis-associated colon cancer. Furthermore, IEC-expressed NLRC4 mediates early innate immune responses against $C$. rodentium via an unknown mechanism independent of IL-1 family cytokine secretion. ${ }^{35}$ Here we show that the caspase-4/11-dependent IECs inflammasome is crucial for IL-18 cytokine maturation and the early innate immune response to EPEC/C. rodentium. Consistently with this, Song-Zhao et al. ${ }^{17}$ recently suggested, based on studies of $\mathrm{Nlrp}^{-/-}$and $\mathrm{Asc}^{-/-}$mice, that early protection to $C$. rodentium infection is mediated by IECs independently of NLRP3 activation. Taken together, our study identifies a fundamental and novel role for the T3SS effector $\mathrm{NleF}$ in the pathogenesis and virulence of $\mathrm{A} / \mathrm{E}$ pathogens through the inhibition of the newly characterized IECs caspase4/11-dependent inflammasome.

\section{METHODS}

Strains, oligonucleotides, plasmids, and antibodies. Strains, plasmids, and primers used in this study are listed in Supplementary Tables S2-S3, respectively. nleF was amplified from EPEC E2348/69 and C. rodentium ICC169 genomic DNA by PCR. Site-directed 
a

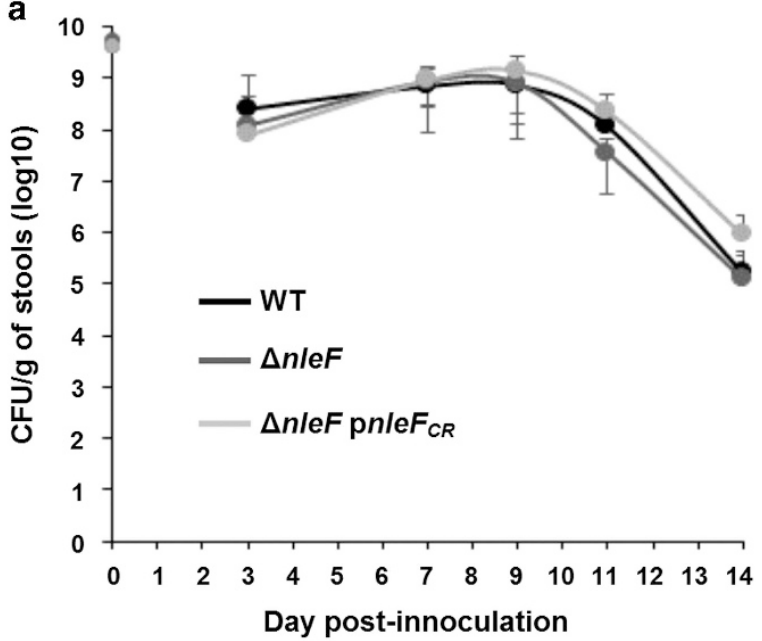

c

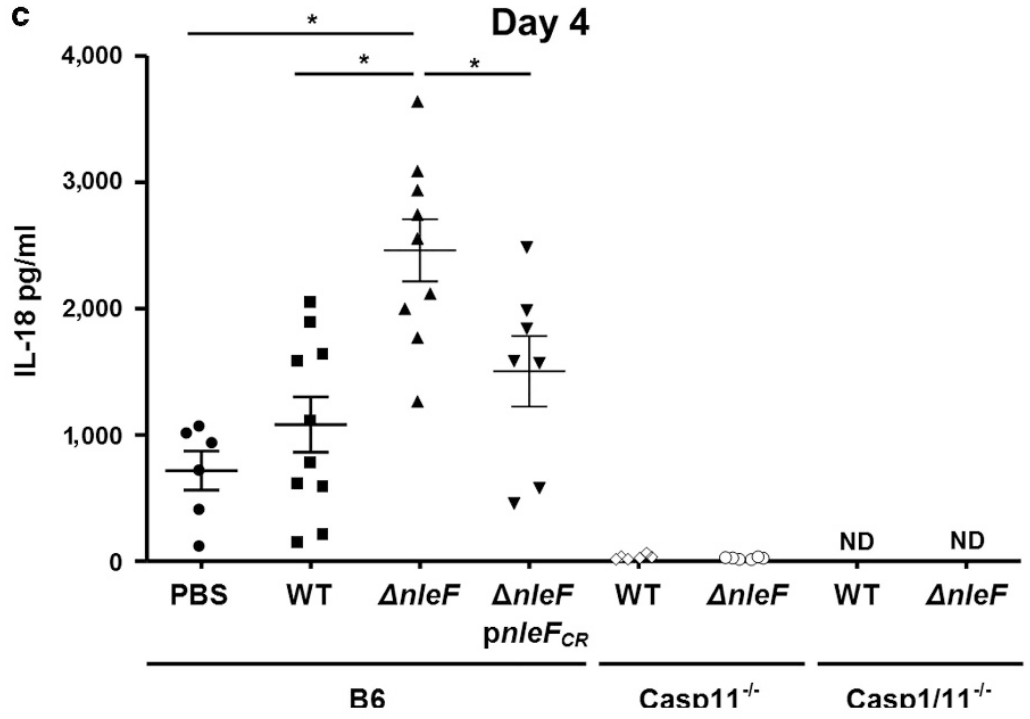

b

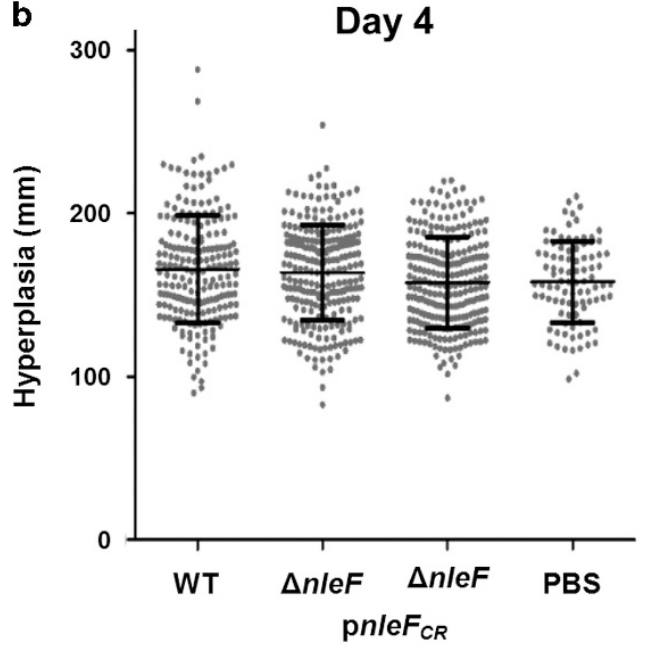

d

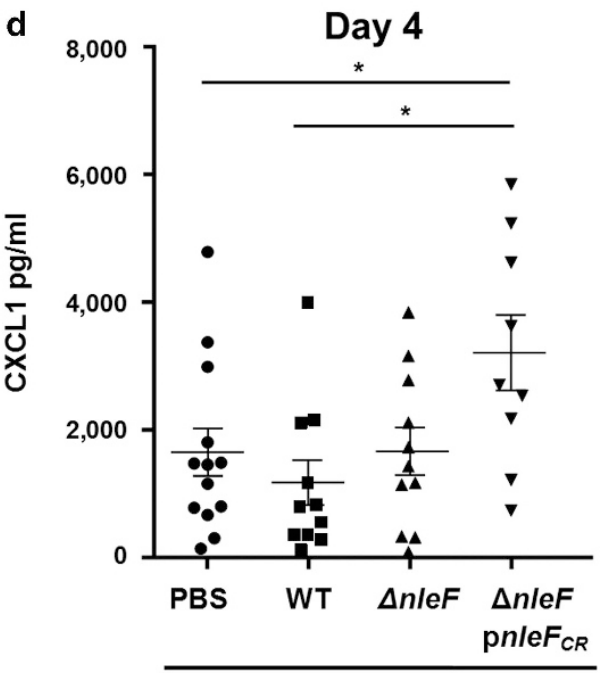

B6

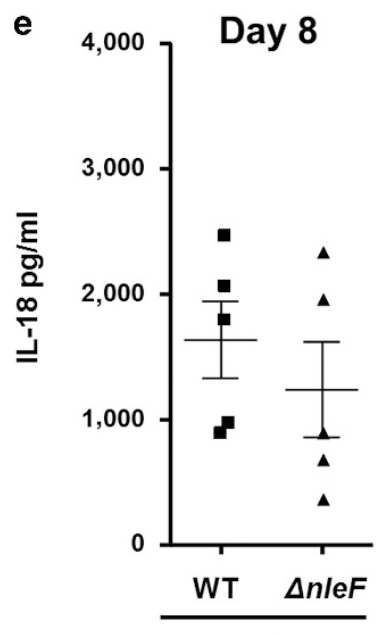

B6

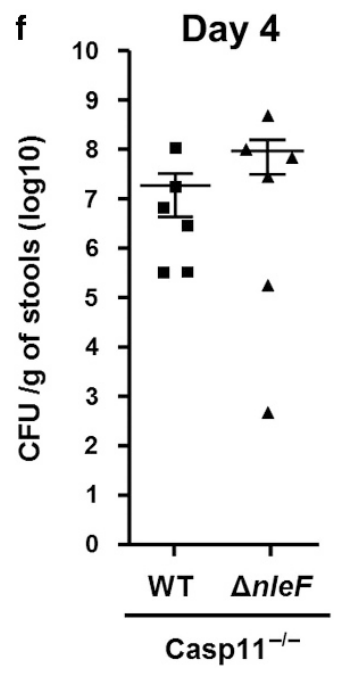

Figure $5 \quad \mathrm{NleF}_{\mathrm{CR}}$ inhibits colonic IL-18 secretion 4 days p.i. WT C. rodentium, C. rodentium $\Delta n l e F$, and the complemented strain ( $\Delta$ nle $F$ pnleFCR) similarly colonized and triggered colonic hypoplasia in C57BL/6 mice (a and b). Each dot in (b) represents an individual measurement of crypt length (from at least 20 measurements per section per mouse), and horizontal bars represent mean values. Significant increase in secreted IL-18, measured by ELISA, was seen

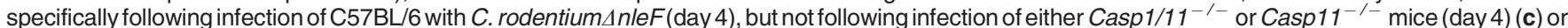
C57BL/6 (day 8) (e). Secreted CXCL1 was found in similar levels, except for the complemented strain, which triggered greater secretion of CXCL1 (d). No

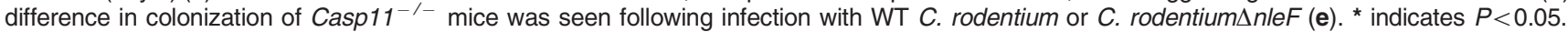


a

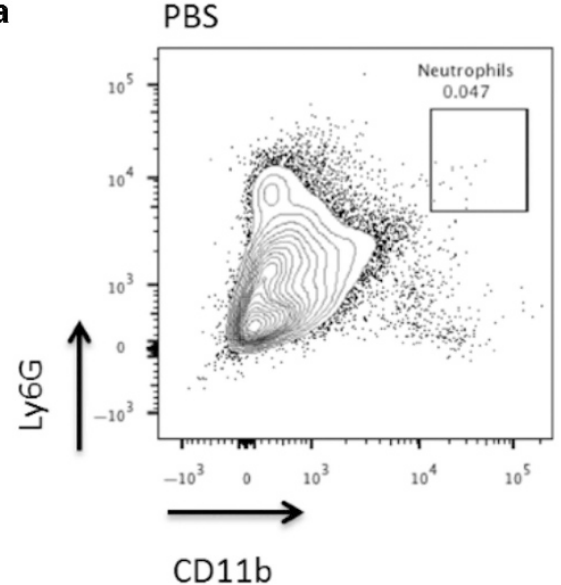

WT

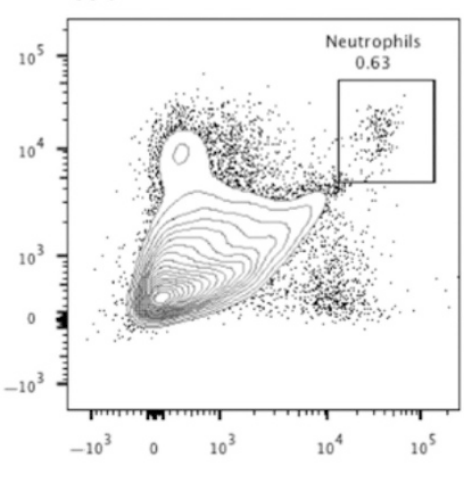

$\Delta n l e F$

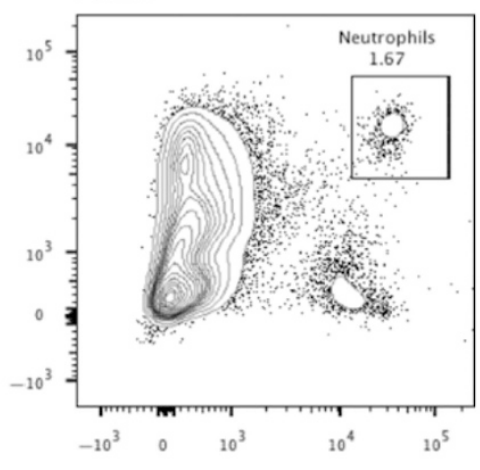

b

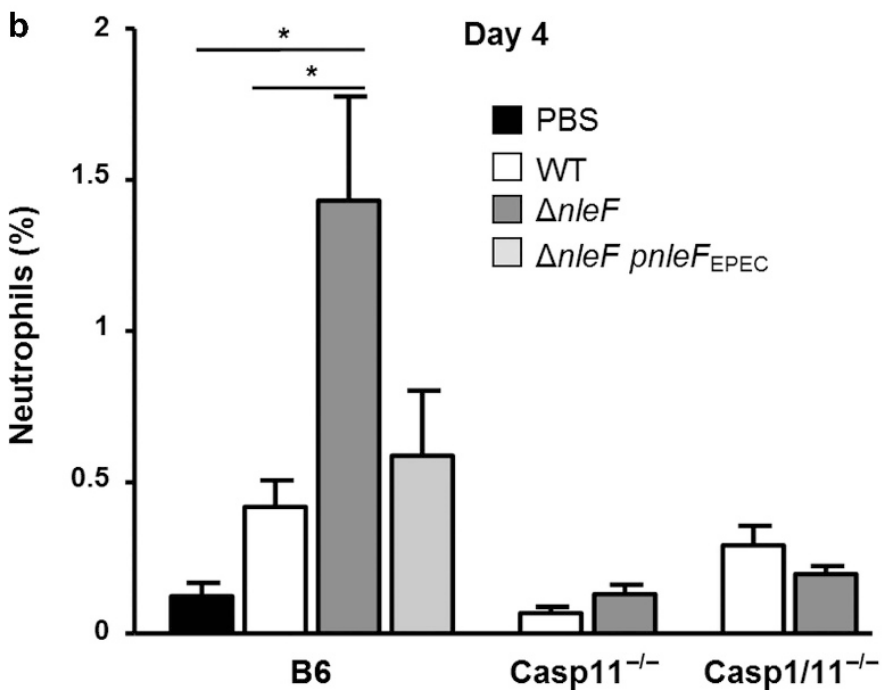

c 8

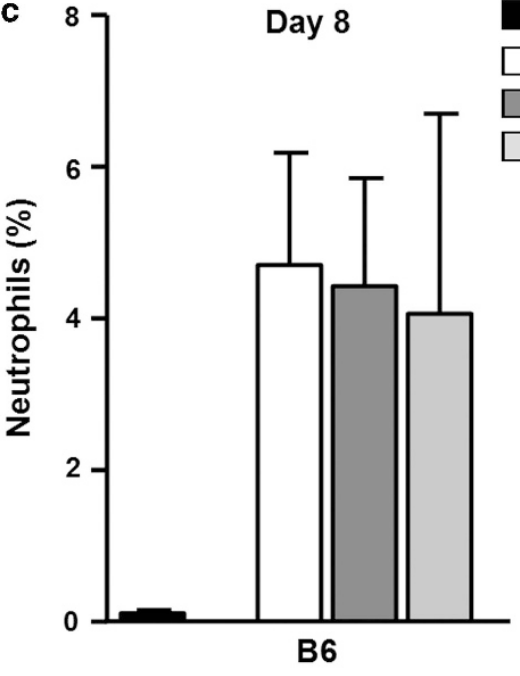

PBS

WT

$\Delta$ nleF

$\Delta$ nleF pnle $F_{\mathrm{EPEC}}$

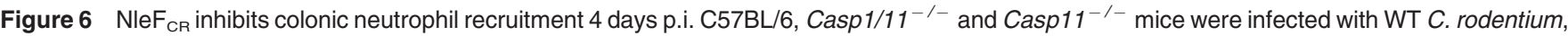
C. rodentium $\triangle$ nleF or complemented $C$. rodentium $\Delta$ nle $F\left(\Delta n l e F\right.$ pnle $\left.F_{\mathrm{CR}}\right)$. (a) Representative image of flow cytometry gating strategy for neutrophils $(\mathrm{CD} 11 \mathrm{~b}+\mathrm{Ly} 6 \mathrm{G}+)$ of control (PBS) and infected C57BL/6 mice. The number of neutrophils $(\mathrm{CD} 11 \mathrm{~b}+\mathrm{Ly} 6 \mathrm{G}+)$ present within the myeloid gate was

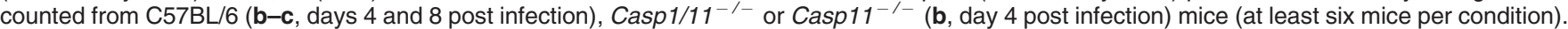
* indicates $P<0.05$

mutagenesis was carried out by inverse PCR using KOD Hot Start polymerase and mismatch primers. All constructs were confirmed by sequencing (GATC Biotech, Konstanz, Germany). For western blot, mouse monoclonal anti-caspase-4 clone 4B9 (sc-56056; Santa Cruz, Heidelberg, Germany), anti- $\alpha$-tubulin clone DM1A (T6199), mouse polyclonal antibody anti-caspase-11 p20 clone A-2 (sc-374615; Santa cruz), and anti-pro-IL-18 (CPTC-IL18-1; DSHB, Iowa City, IA), the rabbit monoclonal anti-IL-18 (PM014; MBL, Woburn, MA), anticaspase-5 (4429; Cell Signalling, Leiden, The Netherlands), and the rabbit polyclonal antibody anti-GFP (Ab290; Abcam, Cambridge, UK) were used as primary antibodies. Horse radish peroxidase-conjugated goat anti-rabbit IgG (Fc fragement; catalog no.111-035-008; Jackson Immunoresearch, West Grove, PA) and horse radish peroxidaseconjugated goat anti-mouse IgG (Fc fragement; catalog no, 115-035008; Jackson Immunoresearch) were used as secondary antibodies.

Retroviral transductions and stable knockdown cell lines. MicroRNA30 based (miR-30; Supplementary Table S1) gene silencing constructs were generated in pMX-CMV-YFP using one-step sequence and ligation independent cloning ${ }^{33}$ following the optimized miR-30E vector design. ${ }^{36}$ Sequences were as follows: CASP4-CGACTGTCCATGACAAGAT; and LacZ (non-targeting negative control) ACGTCGTATTACAACGTCGTGA. The miR-30E plasmids were transfected using Lipofectamine 2000 (Invitrogen), along with the packaging plasmids pVSV-G and pCMV-MMLV-pack ${ }^{37}$ into HEK293E cells to produce a VSV-G pseudotyped retroviral particles for transduction. After $48 \mathrm{~h}$ supernatants were filtered through $0.45 \mu \mathrm{m}$ syringe filters and added directly to pre-seeded Caco-2 TC7 cells. Transduced cells were selected by puromycin (Gibco, Invitrogen, Paisley, UK) at $10 \mu \mathrm{g} \mathrm{ml}^{-1}$ and knockdown was confirmed by western blotting.

EPEC infection, ELISA, and western blotting. Caco-2 TC7 cells (ATCC, Teddington, UK) were seeded at $7.5 \times 10^{4} \mathrm{ml}^{-1}$ and upon reaching confluence ( 7 days) the medium was changed every day for the following 7 days. Before infection the cells were starved for $3 \mathrm{~h}$ in serum-free Dulbecco's Modified Eagle's medium . Monolayers were infected with primed EPEC $^{20}$ at a multiplicity of infection of 1:10 for $3 \mathrm{~h}$. The cells were then washed twice in PBS and the medium was replaced with serum-free Dulbecco's Modified Eagle's medium -high glucose plus penicillin and streptomycin at $100 \mathrm{Uml}^{-1}$ and $100 \mu \mathrm{g} \mathrm{ml}^{-1}$, respectively. After $1 \mathrm{~h}$ cells were washed and either processed for western blot (total IL-18) or incubated for a further $17 \mathrm{~h}$ (secreted caspase-4 and IL-18) with or without Ac-LEVD-CHO (Enzo Lifesciences, Farmingdale, NY). Supernatants were collected, cleared by centrifugation at $13,000 \mathrm{rpm}$ at $4{ }^{\circ} \mathrm{C}$ for $10 \mathrm{~min}$ and precipitated for western blotting with the addition of $10 \%(\mathrm{v} / \mathrm{v})$ trichloroacetic acid for 
$17 \mathrm{~h}$ at $4{ }^{\circ} \mathrm{C}$. The concentration of IL-18 in cell supernatant (MBL) was determined by ELISA according to the manufacturer's protocol.

Cell adhesion and cytotoxicity assays. Caco- 2 TC7 were infected with the WT EPEC, EPEC $\Delta$ nleF and the complemented strain (plasmid encoding $\mathrm{NleF}_{\mathrm{EPEC}}$ ) for $3 \mathrm{~h}$. The monolayers were lysed in $1 \%$ PBS/triton X-100 and EPEC attachment was enumerated by serial dilution on Luria-Bertani (LB)-Agar and calculation of colony forming units.

Supernatants of uninfected cells or cell infected with EPEC for $21 \mathrm{~h}$ were harvested and the level of $\mathrm{LDH}$ release was measured using CytoTox 96 Non-Radioactive Cytotoxicity Assay (Promega, Southampton, UK). As a control for total $\mathrm{LDH}$, cell lysis buffer ( $1 \%$ Triton-X-100/ PBS) was added for $30 \mathrm{~min}$ at $37^{\circ} \mathrm{C}$ directly to the medium and cell layer. Absorbance was measured at $490 \mathrm{~nm}$ using the FluoStar Omega plate reader and results are displayed as percentage of total release corresponding to the $\mathrm{LDH}$ measured in the supernatant divided by the total LDH.

Alternatively, the media was removed and cell layers were incubated in $3.3 \mu \mathrm{g} \mathrm{ml}^{-1}$ PI (Invitrogen) in warm PBS (PI/PBS) for $15 \mathrm{~min}$ and fluorescence was measured at an excitation of $510 \mathrm{~nm}$ and emission of $610 \mathrm{~nm}$ using the FluoStar Omega plate reader. As a control PI/PBS alone was measured or cell lysis buffer $(0.05 \%$ Triton X-100/PBS) supplemented with $3.3 \mu \mathrm{g} \mathrm{ml}{ }^{-1} \mathrm{PI}$ was added for $15 \mathrm{~min}$ at $37^{\circ} \mathrm{C}$. Results are displayed as a percentage of total PI uptake.

Yeast-2-hybrid screen and yeast direct hybrids. A yeast-2-hybrid screen was conducted using pGKBT7-nle $F_{E P E C}$ and the HeLa cell cDNA Library following the manufacturer's Handbook (Clontech). AH109 were co-transformed with pGBT9-bait and pGADT7-prey (Supplementary Table S3) and plated onto Difco Yeast Nitrogen Base without amino acids (SD) agar supplemented with $2 \%$ glucose, $20 \mathrm{mgl}^{-1}$ adenine hemisulfate, $20 \mathrm{mgl}^{-1}$ arginine $\mathrm{HCl}, 20 \mathrm{mgl}^{-1}$ histidine $\mathrm{HCl}$ monohydrate, $30 \mathrm{mgl}^{-1}$ isoleucine, $30 \mathrm{mgl}^{-1}$ lysine $\mathrm{HCl}, 20 \mathrm{mgl}^{-1}$ methionine, $50 \mathrm{mgl}^{-1}$ phenylalanine, $200 \mathrm{mgl}^{-1}$ threonine, $30 \mathrm{mgl}^{-1}$ tyrosine, $20 \mathrm{mgl}^{-1}$ uracil, $150 \mathrm{mgl}^{-1}$ valine and lacking tryptophan and leucine (DDO; Double Drop-out) for selection of transformed clones. Clones positive for both plasmids were re-streaked on to SD DDO and SD QDO /-His/-Ade supplemented with $40 \mathrm{mgl}^{-1} \mathrm{x}$ - $\alpha$-gal (SD QDO) for selection of positive interactions.

Recombinant protein expression and purification. E. coli BL21 Star expressing pET28-NleF EPEC $_{\text {(pICC1659), pET28-NleF }}$ 1-185-EPEC (pICC1660), and pET28-NleF $\mathrm{CR}_{\mathrm{C}}$ (pICC1839) were cultured for $16 \mathrm{~h}$ in $\mathrm{LB}$ at $37^{\circ} \mathrm{C}$ at $200 \mathrm{rpm}$. Bacteria were sub-cultured at 1:100 into $1 \mathrm{~L} \mathrm{LB}$ supplemented with $50 \mu \mathrm{g} \mathrm{ml}^{-1}$ kanamycin and incubated at $37^{\circ} \mathrm{C}$ at $200 \mathrm{rpm}$ until $\mathrm{OD}_{600}$ of $0.4-0.6$. Cultures were then induced with $0.5 \mathrm{~mm}$ isopropyl-beta-D-thiogalactopyranoside for $18 \mathrm{~h}$ at $18^{\circ} \mathrm{C}$. Cells were harvested by centrifugation at $10000 \mathrm{rpm}$ for $20 \mathrm{~min}$ and resuspended in $30 \mathrm{ml}$ ice cold His-lysis buffer ( $20 \mathrm{~mm}$ Tris- $\mathrm{HCl} \mathrm{pH}$ 7.9, $0.5 \mathrm{M} \mathrm{NaCl}$, and $5 \mathrm{~mm}$ Imidazole). The cells were lysed by Emulsiflex following the manufacturer's instructions (Emulsiflex-B15; Avestin, Mannheim, Germany) and centrifuged at $14,000 \mathrm{rpm}$ for a further $30 \mathrm{~min}$ at $4{ }^{\circ} \mathrm{C}$. Supernatant was removed and applied to $5 \mathrm{ml}$ His resin (Novagen, Watford, UK) pre-charged in $5 \mathrm{mM} \mathrm{NiSO}{ }_{4}$ and preequilibrated in His-lysis buffer and rocked at $4{ }^{\circ} \mathrm{C}$ for $1.5 \mathrm{~h}$. Samples were applied to a Poly-Prep Chromatography column (Qiagen) and flow-through was collected. The column was washed twice with $20 \mathrm{ml}$ His-lysis buffer and once in $20 \mathrm{ml}$ wash buffer (Tris- $\mathrm{HCl} \mathrm{pH} \mathrm{7.9,} 0.5 \mathrm{M}$ $\mathrm{NaCl}$, and $60 \mathrm{~mm}$ Imidazole). His-tagged fusion proteins were eluted with $10 \times 1 \mathrm{ml}$ elution buffer (His-lysis buffer supplemented with $1 \mathrm{M}$ Imidazole). Fractions containing His-purified NleF were checked by sodium dodecyl sulfate-polyacrylamide gel electrophoresis and further purified by size exclusion (Akta Prime, GE Healthcare, Little Chalfont, UK) with a Superdex75 column (GE Healthcare; 10/300GL).

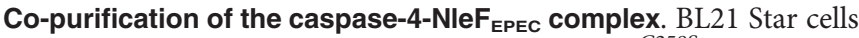
were co-transformed with pACYC-DUET-1-CASP4 ${ }^{\mathrm{C} 258 S}$ His-p20/p10 and pMAL-c2x-nleF $F_{E P E C}$. Bacterial pellets were re-suspended in $20 \mathrm{mM}$
Tris- $\mathrm{HCl} \mathrm{pH} \mathrm{7.4,} 250 \mathrm{~mm} \mathrm{NaCl}$ and lysed by sonication and purified by amylose affinity chromatography. Bacterial lysates were incubated with amylose resin for $1.5 \mathrm{~h}$ at $4{ }^{\circ} \mathrm{C}$ and then washed with $50 \mathrm{ml}$ wash buffer $(20 \mathrm{~mm}$ Tris- $\mathrm{HCl} \mathrm{pH} \mathrm{8.0,250} \mathrm{mM} \mathrm{NaCl}$ and eluted with wash buffer supplemented with $10 \mathrm{~mm}$ maltose. The co-elute was dialysed and then purified further by IMAC talon affinity chromatography, as described previously. ${ }^{38}$ Complex formation was analyzed by size exclusion (Akta prime) with a Superdex200 column (GE Healthcare) using the Gel Filtration Markers Kit for Protein Molecular Weights 12 000-200 000 Da (Sigma-Aldrich, Gillingham, UK) to determine complex size. Size exclusion fractions were verified by sodium dodecyl sulfate-polyacrylamide gel electrophoresis and confirmed by Mass spectrometry.

Caspase activity assays. BL21 star were transformed with pET28aempty, pET28a-CASP4, pET28a-CASP4 C258S or pET28a-Casp11. Soluble lysates at $200 \mu \mathrm{g} / \mathrm{ml}$ were incubated with or without $50 \mu \mathrm{M} \mathrm{Ac-}$ LEVD-AFC (Enzo Life Sciences) in $20 \mathrm{~mm}$ PIPES, $100 \mathrm{~mm} \mathrm{NaCl}$, $10 \mathrm{mM}$ DTT, 1 mM EDTA, $0.1 \%$ CHAPS, $10 \%$ sucrose $\mathrm{pH} 7.2$ or $20 \mathrm{~mm}$ Tris, $250 \mathrm{~mm} \mathrm{NaCl} \mathrm{pH} 7.4$ for caspase-11 and caspase-4, respectively. Purified recombinant His-NleF derivatives were added at varying concentrations from $50 \mathrm{~nm}$ to $1 \mathrm{pM}$. Fluorescence was measured in $5 \mathrm{~min}$ intervals at $37^{\circ} \mathrm{C}$ using an excitation of $410 \mathrm{~nm}$ and emission of $520 \mathrm{~nm}$ using the FLUOstar Omega plate reader (BMG Labtech, Aylesbury, UK).

Construction of $\boldsymbol{C}$. rodentium mutant. C. rodentium strain ICC169 $\Delta n l e F$ (ICC1129) was generated using a modified version of the lambda red-based mutagenesis system. ${ }^{39}$ In brief, the $n l e F$ gene and its flanking regions were PCR-amplified from WT C. rodentium ICC169 genomic DNA using the primers pair NleF-up-Fw/NleF-down-Rv and cloned into pC-Blunt-TOPO vector (Invitrogen). The $n l e F$ gene was then excised using inverse PCR (primers NleF-up-Rv-BamHI/NleFdown-Fw-BamHI) and the resulting linear product was BamHI digested, allowing insertion of the non-polar $a p h T,{ }^{40}$ cassette, resulting in plasmid pICC1674. After verifying for correct orientation of the kanamycin cassette, the insert was PCR-amplified using NleF-up-Fw and NleF-down-Rv primers. The PCR products were electroporated into wild-type $C$. rodentium expressing the lambda red recombinase from pKD46 plasmid. The deletion was confirmed by PCR and DNA sequencing among the kanamycin resistant clones (primers NleF-upFw-check and NleF-down-Fw-check).

Oral infection of mice. Pathogen-free female C57BL/6 mice were either purchased from Charles River (Harlow, England, UK) or sourced from BIME Institut Pasteur. Casp $1 / 11^{-}-$mice were generously provided by Bernhard Ryffel (TAAM-CDTA, Orelans, France) and Casp $11^{-}$- were generously provided by Mohamed Lamkanfi (Ghent University, Belgium). All animals were housed in individually high-efficiency particulate arrestance -filtered cages with sterile bedding and free access to sterilized food and water. Independent infection experiments for wild-type C57BL/6, Casp1/11 ${ }^{-/-}$ and Casp $11^{-/-}$mice were performed using 3-8 mice per group. Mice were infected and followed for shedding as described. ${ }^{41}$ In brief, mice were infected via oral gavage with $10^{9}$ WT $C$. rodentium or $C$. rodentium $\Delta n l e F$ as described previously. For control, mice were gavaged with sterile PBS. The number of viable bacteria used as inoculum was determined by retrospective plating onto LB agar containing antibiotics. Stool samples were recovered aseptically at various time points after inoculation and the number of viable bacteria per gram of stool was determined by plating onto LB agar. ${ }^{41}$

Sample collection and colonic crypt hyperplasia measurement. Segments of the terminal colon $(0.5 \mathrm{~cm})$ of each mouse were collected, flushed, and fixed in 10\% neutral buffered formalin. Formalin fixed tissues were then processed, paraffin-embedded, sectioned at $5 \mu \mathrm{m}$ and stained with H\&E (haematoxylin and eosin) using standard techniques. H\&E-stained tissues were evaluated for colonic crypt 
hyperplasia microscopically without knowledge of the treatment condition used in the study and the length of at least 100 well-oriented crypts from each section from all of the mice per treatment group ( $n=4-6)$ were evaluated. H\&E-stained tissues were imaged with an Axio Lab.A1 microscope (Carl Zeiss MicroImaging,Germany), images were acquired using an Axio Cam ERc5s color camera, and computer-processed using AxioVision (Carl Zeiss MicroImaging, Berlin, Germany).

Sample collection for cytokine analysis and flow cytometry. Isolation of colonic cells and flow cytometry were performed as described. ${ }^{41}$ After a PBS wash, the 5 th $\mathrm{cm}$ of the distal colon was incubated in Rosewell Park Memorial Institute medium containing penicillin, streptomycin, gentamicin, and fetal bovine serum at $37^{\circ} \mathrm{C}$ for $24 \mathrm{~h}$. The concentrations of IL-18 (eBioscience, San Diego, CA, \#BMS618/3), IL-1 $\beta$, and KC (CXCL1; R\&D Systems, Minneapolis, $\mathrm{MN}$ ) were determined by ELISA according to the manufacturer's protocols.

Statistics. All data were analyzed using GraphPad Prism software, using the Mann-Whitney test and represented as the mean \pm s.e.m. or s.d. A $P$-value $<0.05(P<0.05)$ was considered statistically significant.

SUPPLEMENTARY MATERIAL is linked to the online version of the paper at http://www.nature.com/mi

\section{ACKNOWLEDGMENTS}

We thank Guy Frankel for making the $\mathrm{CR} \Delta$ nle $F$ mutant. We are grateful to $\mathrm{Dr}$ Bernhard Ryffel (TAAM-CDTA, Orelans, France) and Dr Mohamed (Lamkanfi, VIB Inflammation Research Center, Ghent University, Belgium) for providing us with the Casp $1 / 11^{-/-}$and Casp $11^{-/-}$mice. This project was supported by grants to GF from the Biotechnology and Biological Sciences Research Council (BBSRC), the Wellcome Trust and the Medical Research Council (MRC). ARS acknowledges funds from the Royal Society (RG130811) and the Wellcome Trust (108246/Z/15/Z). J.P.D. and N.S. are supported by grants from the Institut Pasteur, Inserm, and Danone.

\section{AUTHOR CONTRIBUTIONS}

M.A.P., V.F.P., N.S., and C.N.B. - plan and conducted experiments and wrote the paper. M.H., O.K., and J.S.G. - plan and conducted experiments. J.P.D.S., A.R.S., and G.F. - plan experiments and wrote the paper.

\section{DISCLOSURE}

The authors declare no conflict of interest.

c) 2017 Society for Mucosal Immunology

\section{REFERENCES}

1. Croxen, M.A., Law, R.J., Scholz, R., Keeney, K.M., Wlodarska, M. \& Finlay, B.B. Recent advances in understanding enteric pathogenic Escherichia coli. Clin. Microbiol. Rev. 26, 822-880 (2013).

2. Collins, J.W. et al. Citrobacter rodentium: infection, inflammation and the microbiota. Nat. Rev. Microbiol. 12, 612-623 (2014).

3. Baruch, K. et al. Metalloprotease type III effectors that specifically cleave JNK and NF-kappaB. EMBO J. 30, 221-231 (2011).

4. Zhang, L. et al. Cysteine methylation disrupts ubiquitin-chain sensing in NF-kB activation. Nature 481, 204-208 (2011).

5. Pearson, J.S., Riedmaier, P., Marchès, O., Frankel, G. \& Hartland, E.L. A type III effector protease $\mathrm{NleC}$ from enteropathogenic Escherichia coli targets NF-?B for degradation. Mol. Microbiol. 80, 219-230 (2011).

6. Li, S. et al. Pathogen blocks host death receptor signalling by arginine GlcNAcylation of death domains. Nature 501, 242-246 (2013).

7. Pham, T.H., Gao, X., Singh, G. \& Hardwidge, P.R. Escherichia coli virulence protein $\mathrm{NleH} 1$ interaction with the $v$-Crk sarcoma virus CT10 oncogene-like protein (CRKL) governs $\mathrm{NleH} 1$ inhibition of the ribosomal protein S3 (RPS3)/nuclear factor kappaB (NF-kappaB) pathway. J. Biol. Chem. 288, 34567-34574 (2013).
8. von Moltke, J., Ayres, J.S., Kofoed, E.M., Chavarria-Smith, J. \& Vance, R.E. Recognition of bacteria by inflammasomes. Annu. Rev. Immunol. 31, 73106 (2013).

9. Eldridge, M.J. \& Shenoy, A.R. Antimicrobial inflammasomes: unified signalling against diverse bacterial pathogens. Curr. Opin. Microbiol. 23, 32-41 (2015).

10. Shi, J. et al. Inflammatory caspases are innate immune receptors for intracellular LPS. Nature 514, 187-192 (2014).

11. Kayagaki, N. et al. Non-canonical inflammasome activation targets caspase-11. Nature 479, 117-121 (2011).

12. Casson, C.N. et al. Human caspase-4 mediates noncanonical inflammasome activation against gram-negative bacterial pathogens. Proc. Natl. Acad. Sci. USA 112, 6688-6693 (2015).

13. Rathinam, V.A. et al. TRIF licenses caspase-11-dependent NLRP3 inflammasome activation by gram-negative bacteria. Cell 150, 606-619 (2012).

14. Knodler, L.A. et al. Noncanonical inflammasome activation of caspase-4/ caspase-11 mediates epithelial defenses against enteric bacterial pathogens. Cell. Host Microbe 16, 249-256 (2014).

15. Hemrajani, C., Berger, C.N., Robinson, K.S., Marches, O., Mousnier, A. \& Frankel, G. NleH effectors interact with Bax inhibitor-1 to block apoptosis during enteropathogenic Escherichia coli infection. Proc. Natl. Acad. Sci. USA 107, 3129-3134 (2010).

16. Liu, Z. et al. Role of inflammasomes in host defense against Citrobacter rodentium infection. J. Biol. Chem. 287, 16955-16964 (2012).

17. Song-Zhao, G.X., Srinivasan, N., Pott, J., Baban, D., Frankel, G. Maloy, K.J. Nlrp3 activation in the intestinal epithelium protects against a mucosal pathogen. Mucosal Immunol 7, 763-774 (2014).

18. Yen, H., Sugimoto, N. \& Tobe, T. Enteropathogenic Escherichia coli uses NleA to inhibit NLRP3 inflammasome activation. PLOS Pathog. 11, e1005121 (2015).

19. Blasche, S. et al. The E. coli effector protein NleF Is a caspase inhibitor. PLoS One 8, e58937 (2013).

20. Pallett, M.A., Berger, C.N., Pearson, J.S., Hartland, E.L. \& Frankel, G. The type III secretion effector NleF of enteropathogenic Escherichia coli activates NF-kappaB early during infection. Infect. Immun. 82, 4878-4888 (2014).

21. Dinarello, C.A. \& Fantuzzi, G. Interleukin-18 and host defense against infection. J. Infect. Dis. 187 (Suppl 2), S370-S384 (2003).

22. Brodsky, I.E. et al. A Yersinia effector protein promotes virulence by preventing inflammasome recognition of the type III secretion system. Cell. Host Microbe 7, 376-387 (2010).

23. Hagar, J.A., Powell, D.A., Aachoui, Y., Ernst, R.K. \& Miao, E.A. Cytoplasmic LPS activates caspase-11: implications in TLR4-independent endotoxic shock. Science 341, 1250-1253 (2013).

24. Kobayashi, T. et al. The Shigella OspC3 effector inhibits caspase-4, antagonizes inflammatory cell death, and promotes epithelial infection. Cell. Host Microbe 13, 570-583 (2013).

25. Gurung, P. et al. Toll or interleukin-1 receptor (TIR) domain-containing adaptor inducing interferon-beta (TRIF)-mediated caspase-11 protease production integrates Toll-like receptor 4 (TLR4) protein- and NIrp3 inflammasome-mediated host defense against enteropathogens. J. Biol. Chem. 287, 34474-34483 (2012).

26. Verri, W.A. Jr et al. IL-15 mediates antigen-induced neutrophil migration by triggering IL-18 production. Eur. J. Immunol. 37, 3373-3380 (2007).

27. Canetti, C.A., Leung, B.P., Culshaw, S., Mclnnes, I.B., Cunha, F.Q. \& Liew, F.Y. IL-18 enhances collagen-induced arthritis by recruiting neutrophils via TNF-alpha and leukotriene B4. J. Immunol. 171, 10091015 (2003).

28. Dupaul-Chicoine, J. et al. Control of intestinal homeostasis, colitis, and colitis-associated colorectal cancer by the inflammatory caspases. Immunity 32, 367-378 (2010).

29. Daig, R. et al. Human intestinal epithelial cells secrete interleukin-1 receptor antagonist and interleukin-8 but not interleukin-1 or interleukin-6. Gut 46, 350-358 (2000).

30. Basu, R. et al. Th22 cells are an important source of IL-22 for host protection against enteropathogenic bacteria. Immunity 37, 1061-1075 (2012).

31. Spehlmann, M.E., Dann, S.M., Hruz, P., Hanson, E., McCole, D.F. \& Eckmann, L. CXCR2-dependent mucosal neutrophil influx protects against colitis-associated diarrhea caused by an attaching/effacing lesion-forming bacterial pathogen. J. Immunol. 183, 3332-3343 (2009). 


\section{ARTICLES}

32. Elinav, E., Henao-Mejia, J. \& Flavell, R.A. Integrative inflammasome activity in the regulation of intestinal mucosal immune responses. Mucosal Immunol 6, 4-13 (2013).

33. Wlodarska, M. et al. NLRP6 inflammasome orchestrates the colonic hostmicrobial interface by regulating goblet cell mucus secretion. Cell 156, 1045-1059 (2014).

34. Allen, I.C. Non-inflammasome forming NLRs in inflammation and tumorigenesis. Front. Immunol 5, 169 (2014).

35. Nordlander, S., Pott, J. \& Maloy, K.J. NLRC4 expression in intestinal epithelial cells mediates protection against an enteric pathogen. Mucosal Immunol 7, 775-785 (2014).

36. Fellmann, C. et al. An optimized microRNA backbone for effective singlecopy RNAi. Cell. Rep 5, 1704-1713 (2013).

37. Shenoy, A.R. et al. GBP5 promotes NLRP3 inflammasome assembly and immunity in mammals. Science 336, 481-485 (2012).
38. Kotik-Kogan, O., Valentine, E.R., Sanfelice, D., Conte, M.R. \& Curry, S. Structural analysis reveals conformational plasticity in the recognition of RNA 3' ends by the human La protein. Structure 16 , 852-862 (2008).

39. Datsenko, K.A. \& Wanner, B.L. One-step inactivation of chromosomal genes in Escherichia coli K-12 using PCR products. Proc. Natl. Acad. Sci. USA 97, 6640-6645 (2000).

40. Galan, J.E., Ginocchio, C. \& Costeas, P. Molecular and functional characterization of the Salmonella invasion gene invA: homology of InvA to members of a new protein family. J. Bacteriol. 174, 43384349 (1992).

41. Crepin, V.F., Habibzay, M., Glegola-Madejska, I., Guenot, M., Collins, J.W. \& Frankel, G. Tir triggers expression of CXCL1 in enterocytes and neutrophil recruitment during citrobacter rodentium infection. Infect. Immun. 83, 3342-3354 (2015). 